\title{
El conocimiento didáctico-matemático: una propuesta de evaluación de tres de sus facetas ${ }^{1}$
}

The mathematical didactic knowledge: a proposal for evaluation of three of its facets

O conhecimento didático matemática: uma proposta de avaliação de três de suas facetas

Recibido: mayo de 2013

Aceptado: agosto de 2013
Walter F. Castro $\mathrm{G}^{2}$

Luis R. Pino-Fan ${ }^{3}$

Hilduara Velasquez $\mathrm{E}^{4}$

\section{Resumen}

En esta comunicación se presentan algunos criterios tenidos en cuenta para el diseño de un cuestionario para evaluar tres facetas del conocimiento matemático para la enseñanza de la derivada: el conocimiento común del contenido, el conocimiento especializado y el conocimiento ampliado. Así mismo se presenta una tarea propuesta en el cuestionario aplicado a estudiantes de las licenciaturas en Básica Matemáticas y Matemáticas -Física de la Universidad de Antioquia, Colombia.

Palabras clave: Formación de maestros. Conocimiento del maestro. Enfoque Ontosemiótico. Faceta Epistémica. Matemáticas escolares. Cálculo. Derivada. Evaluación. Instrumentos de evaluación.

\section{Abstract}

In this paper we present some criteria taken into account for the design of a questionnaire to assess three facets of mathematical knowledge for teaching the derivative: common content knowledge, specialized knowledge and expanded awareness. It also presents a task proposed in the questionnaire given to students of the degrees in Basic Mathematics and MathematicsPhysics, University of Antioquia, Colombia.

Keywords: Training of teachers. Knowledge of the teacher. Ontosemiotic approach. Epistemic Facet. School mathematics. Calculation. Derivative. Evaluation. Assessment instruments.

\section{Resumo}

Neste artigo, apresentamos alguns critérios tidos em conta para a elaboração de um questionário para avaliar três aspectos do conhecimento matemático para o ensino do derivado: o conhecimento do conteúdo comum, conhecimento especializado e expansão da consciência. Apresenta, também, uma tarefa proposta no questionário aplicado aos alunos dos graus

1 Artículo de Investigación.

2 Universidad de Antioquia. Medellin, Colombia. Contacto: wfcastro82@gmail.com

3 Universidad de Granada. Contacto: luispino23@gmail.com

4 Universidad de Antioquia. Medellin, Colombia. Contacto: sarcavelasquez@gmail.com 
em Matemática Básica e Matemática-Física da Universidade de Antioquia, Colômbia.

Palavras-chave: Formação de professores. O conhecimento do professor. Abordagem ontosemiótico. Facet epistêmica. A matemática escolar. Cálculo. Derivativo. Avaliação. Instrumentos de avaliação.

\section{Antecedentes}

La formación matemática y didáctica de los futuros maestros constituye un campo de investigación que tiene mucha actividad en la comunidad de investigadores en Matemática Educativa. En Colombia existe un acentuado interés por parte del Ministerio de Educación de promover la investigación sobre la calidad de la formación tanto de maestros en ejercicio como de maestros en formación. Una de las razones es que el desarrollo del pensamiento y de las competencias matemáticas de los alumnos depende de manera esencial de la formación de sus maestros.

Las reflexiones y recomendaciones de Shulman (1986) y las investigaciones de Ball (2000); Ball, Lubienski \& Mewborn (2001); Hill, Ball \& Schilling (2008), avanzan hacia la caracterización de los componentes del complejo de conocimientos que un maestro debería tener para desarrollar eficazmente su práctica y facilitar el aprendizaje de los estudiantes.

Una manera de profundizar en los conocimientos requeridos para la enseñanza de la derivada es mediante el diseño y aplicación de pruebas que consideren los diversos niveles que componen el conocimiento matemático para la enseñanza (Godino, 2009). En esta comunicación se informa sobre los criterios usados para el diseño de un cuestionario que pretende evaluar tres niveles del conocimiento didáctico para la enseñanza: el conocimiento común del contenido, el conocimiento ampliado y especializado. Así mismo se presenta un ejercicio incluido en el cuestionario y se presentan resultados preliminares de una prueba piloto que se realizó en la Facultad de Educación de la Universidad de Antioquia.

\section{Marco teórico y metodología}

Para el desarrollo de este trabajo, hemos considerado el modelo del conocimiento didáctico-matemático (CDM) (Godino, 2009) propuesto dentro del marco del Enfoque Ontosemiótico del conocimiento y la instrucción matemática (Godino, Batanero \& Font, 2007). Dicho modelo del CDM incluye seis facetas o dimensiones del conocimiento didáctico-matemático para la enseñanza y el aprendizaje de temas específicos. De las seis facetas, en este trabajo, utilizamos dos:

Epistémica. Distribución, a lo largo del tiempo de enseñanza, de los componentes del significado institucional implementado (problemas, lenguajes, procedimientos, definiciones, propiedades, argumentos).

Cognitiva. Desarrollo de los significados personales (aprendizajes).

Para cada una de estas facetas se contemplan, a su vez, cuatro niveles (prácticas matemáticas y didácticas; configuraciones de objetos y procesos; normas y meta-normas e idoneidad) que permiten el análisis del CDM del maestro de acuerdo con el tipo de información requerida para la toma de decisiones instruccionales. En este reporte solo se da cuenta del primer y del segundo nivel:

Prácticas matemáticas y didácticas. Descripción de las acciones realizadas para resolver las tareas matemáticas propuestas para contextualizar los contenidos y promover el aprendizaje. También se describen las líneas generales de actuación del docente y discentes. 
Configuraciones de objetos y procesos (matemáticos y didácticos). Descripción de objetos y procesos matemáticos que intervienen en la realización de las prácticas, así como los que emergen de ellas. La finalidad de este nivel es describir la complejidad de los objetos y significados de las prácticas matemáticas y didácticas. Tal complejidad es un elemento explicativo tanto de los conflictos de significado como de la progresión del aprendizaje.

La investigación se inscribe dentro de un enfoque metodológico de tipo mixto (Johnson \& Onwuegbuzie, 2004), puesto que se trata de un estudio de tipo exploratorio en el que se considera la observación de variables cuantitativas (grado de corrección de los ítems: respuestas correctas, parcialmente correctas e incorrectas) y cualitativas (tipo de resolución propuestas por los futuros maestros). Para el estudio cualitativo utilizamos la técnica de análisis denominada análisis semiótico (Godino, 2002), la cual permite describir de manera sistemática tanto la actividad matemática realizada por los futuros maestros al resolver problemas, como los objetos matemáticos primarios (elementos lingüísticos, conceptos/definiciones, proposiciones/propiedades, procedimientos y argumentos) que intervienen en las prácticas que permiten su resolución (Godino et al., 2007).

Sujetos y Contexto. La prueba se aplicó a una muestra de 45 estudiantes de los últimos cursos (sexto y octavo semestre) de la Licenciatura en Matemáticas y Física y Licenciatura en Básica Matemática que se imparte en la Facultad de Educación de la Universidad de Antioquia. Se debe indicar que los estudiantes en nuestro estudio, suelen desempañarse como maestros en los niveles de primaria, secundaria, bachillerato o universitario. Los 45 estudiantes a los que se les aplicó el cuestionario habían cursado cálculo diferencial y han cursado asignaturas relacionadas con el análisis matemático, con las matemáticas y con su didáctica. Los estudiantes trabajaron de manera individual.
El Cuestionario. Que hemos denominado Cuestionario sobre la Faceta Epistémica del Conocimiento Didáctico-Matemático de la Derivada (Cuestionario FE-CDM-Derivada) consta, inicialmente, de catorce tareas y fue diseñado con base en el modelo para la evaluación y desarrollo del conocimiento didáctico-matemático propuesto por Godino (2009).

El cuestionario se centra, fundamentalmente, en la evaluación de tres tipos de conocimiento: conocimiento común, conocimiento especializado y conocimiento ampliado (Ball et al., 2001; Hill et al., 2008). En el proceso de construcción del cuestionario se consideraron tres criterios para la selección de las tareas que lo conforman. El primer criterio considera que las tareas deben proporcionar información sobre el grado de ajuste del significado personal de los futuros maestros respecto del significado global u holístico del objeto derivada (Pino-Fan, Godino \& Font, 2011). Para lograrlo, se incluyeron ítems que activan distintos sentidos para el objeto derivada (pendiente de la recta tangente, razón instantánea de cambio y tasa instantánea de variación).

El segundo criterio fue que los ítems seleccionados respondan a los diferentes tipos de representaciones activados en los tres subprocesos, que según Font (1999), intervienen en el cálculo de la función derivada:

1. Traducciones y conversiones entre las distintas formas de representar

2. El paso de una representación de a una forma de representación. de

3. Traducciones y conversiones entre las distintas formas de representar.

En este sentido, las tareas incluidas en el cuestionario ponen en juego los diferentes tipos de representaciones que intervienen en estos tres subprocesos: descripción verbal, gráfica, fórmula y tabular; tanto para la función como para su derivada. El 
tercer criterio considera la inclusión de tres tipos de tareas: (1) aquellas que piden poner en juego el conocimiento común del contenido (este conocimiento hace referencia a la resolución de tareas matemáticas que se proponen usualmente en el currículo del bachillerato); (2) aquellas que requieren del conocimiento especializado (usar distintas representaciones, distintos significados parciales de un objeto matemático, resolver el problema mediante diversos procedimientos, dar diversas argumentaciones válidas, identificar los conocimientos puestos en juego durante la resolución de una tarea matemática, etc.); y (3) aquellas que requieren del conocimiento ampliado (generalizar tareas sobre conocimiento común o especializado y/o realizar conexiones con objetos matemáticos más avanzados en el currículo).

La tarea. A continuación se presenta una de las tareas incluidas en el cuestionario CDM-derivada. La Tarea 12 (Figura 1) proporciona información sobre el conocimiento ampliado de los maestros, ya que se trata de una tarea que los futuros maestros deben valorar e identificar los conflictos de significado manifestados en las soluciones de los estudiantes.

Figura: Tarea 12 del cuestionario sobre la derivada

Tarea 12: A continuación se muestra una tarea sobre derivadas propuesto a estudiantes de cálculo I. Posteriormente se muestran soluciones dadas por los estudiantes. Usted debe tanto valorar cada solución, indicando si la considera correcta o incorrecta, y debe identificar los supuestos "matemáticos" que indujeron al estudiante a dar tal respuesta:

Problema: Considere la función cuya ley de asignación está dada por $\_$. Calcular su derivada con respecto a $\mathrm{x}$.

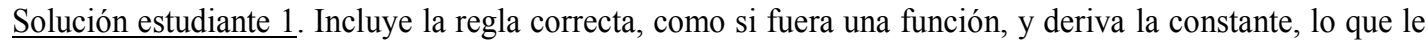
da cero, y al final le da cero en su respuesta

$$
\begin{aligned}
& f^{\prime}(x)=3 \cdot \cos ^{2}\left(\frac{3 \pi}{8}\right) \cdot\left(-\operatorname{sen}\left(\frac{3 \pi}{8}\right)\right) \cdot 0 \\
& \text { Pues la derivada de } \frac{3 \pi}{8} \text { es cero. }
\end{aligned}
$$

Solución estudiante 2. Afirma que es una constante y la derivada da cero.

$$
\begin{aligned}
& F(x)=\left(\cos \frac{3 \pi}{8}\right)^{3} \text { es una constante } \\
& \text { y so derivada es cero. }
\end{aligned}
$$

$\underline{\text { Solución estudiante } 3}$. Deriva como si fuera una regla de la cadena pero no le da cero

$$
f^{\prime}(x)=3\left[\cos \left(\frac{3 \frac{\pi}{2}}{4}\right)\right]^{2} \cdot\left[-\operatorname{sen}\left(\frac{3 \frac{\pi}{2}}{4}\right)\right] \cdot \frac{3 \frac{\pi}{2}}{4}
$$

Fuente: Elaboración propia 


\section{Resultados y discusión}

Por limitaciones de espacio no se presentan aquí los resultados preliminares, lo cual se hará durante la presentación. Sin embargo se puede adelantar que existen resultados mixtos en relación con la manifestación del conocimiento en los tres niveles por parte de los estudiantes. Parece que, tanto para los futuros licenciados en matemática y física como para los licenciados en básica matemática, hay consistencia en los resultados para la faceta "conocimiento común del contenido" pero no así para las otras dos facetas. Los estudiantes recurren en primer lugar a la acepción de la derivada de una función en un punto como pendiente de la recta tangente a la gráfica de una función en un punto, en segundo lugar a la derivada como razón instantánea de cambio. Los cambios de registro requeridos para resolver algunas tareas han probado ser difíciles para los futuros maestros.

\section{Referencias}

Ball, D. L. (2000). Bridging practices: Intertwining content and pedagogy in teaching and learning to teach. Journal of Teacher Education, 51, 241-247.

Ball, D. L., Lubienski, S. T., \& Mewborn, D. S. (2001). Research on teaching mathematics: The unsolved problem of teachers' mathematical knowledge. En V. Richardson (Ed.), Handbook of research on teaching (4th ed., pp. 433-456). Washington, DC: American Educational Research Association.
Font, V. (2000). Procediments per obtenir expressions simbòliques a partir de gràfiques. Aplicacions a la derivada. Tesis doctoral no publicada, Universitat de Barcelona.

Godino, J.D. (2009). Categorías de análisis de los conocimientos del profesor de matemáticas. Unión, Revista Iberoamericana de Educación Matemática, 20, 13-31.

Godino, J. D., Batanero, C., \& Font, V. (2007). The onto-semiotic approach to research in mathematics education. ZDM. The International Journal on Mathematics Education, 39(1), 127-135.

Hill, H. C., Ball, D. L., \& Schlling, S. G. (2008). Unpacking pedagogical content knowledge of students. Journal for Research in Mathematics Education, 39, 372-400.

Johnson, R.B., \& Onwuegbuzie, A. (2004). Mixed methods research: a research paradigm whose time has come. Educational Research, 33(7), 14-26.

Pino-Fan, L., Godino, J.D., \& Font, V. (2011). Faceta epistémica del conocimiento didáctico-matemático sobre la derivada. Educação Matematica Pesquisa, 13(1), 141-178.

Shulman, L. S. (1986). Those who understand: Knowledge growth in teaching. Educational Researcher, 15(2), 4-14. 